\title{
Führungsethik Sozialer Marktwirtschaft Ein systematischer Leitfaden für eine Mikro-Marko-Perspektive
}

Elmar Nass

Received 17 May 2017; Accepted 19 September 2017

Zusammenfassung: Ein Leitfaden wird als Modell vorgestellt, mit dessen Hilfe Führungsethik von Führungstheorie unterschieden und eine an den Wertegrundlagen Sozialer Marktwirtschaft orientierte Führungsethik identifiziert werden kann. Theoretische Konzepte lassen sich mit dieser Makro-Mikro-Perspektive daraufhin überprüfen, ob sie einen Verantwortungsdualismus von Effizienz- und Humanziel in der Führungskultur schlüssig bestimmen. Mithilfe von vorgeschlagenen Prinzipien und Perspektiven kann so in vier Schritten eine Führungsethik in der normativen Linie Sozialer Marktwirtschaft von davon abweichenden Modellen unterschieden werden.

(c) De Gruyter Open Sp. z 0.0.

\section{Darum dieser Leitfaden}

\subsection{Darum ein Leitfaden für Führungsethik}

Der vorliegende Beitrag will mit der Vorstellung eines Leitfadens für werteorientierte Unternehmen, Unternehmer und andere Führungskräfte eine Hilfe sein, im Dschungel von angepriesenen Führungsethiken die Geister unterscheiden zu können. Hierzu gehört es, zunächst einmal Führungsethik als Ethik identifizieren zu können. Dafür wird der Frage nachgegangen, was überhaupt eine Führungsethik ausmacht und was sie von anderen Führungstheorien und von Handwerkskoffern wesentlich unterscheidet. Anschließend wird eine Hilfe angeboten, die Grundideen der verschiedenen Ethikmodelle mithilfe eines Leitfadens einander gegenüberzustellen. Dieser ist für Unternehmen eine Orientierungshilfe für die Entscheidung, welche Führungsethik stringent zu den Werten der eigenen Unternehmensphilosophie passt und in eine Führungsmoral als gelebter Kultur umgesetzt werden soll. Das erfordert zwei Schritte. Zuerst muss bei den angepriesenen Modellen geklärt werden, welches davon überhaupt zu Recht den Namen Ethik trägt. In einem zweiten Schritt kann dann die Stringenz von angebotenen Ethikkonzepten mit den normativen Zielen des Unternehmens überprüft werden.

Wenn ein Unternehmen sich dazu entschließt, eine in sich stimmige Kultur der Führungsmoral auf Grundlage einer systematischen Führungsethik zu kultivieren, dann mögen bei einem erfolgreichen mittelständischen Handwerksunternehmen in Familienbesitz in der Auswahl des Ethikkonzeptes womöglich andere Prioritäten gelten als für einen gemeinnützigen christlichen Träger der Wohlfahrtspflege oder für eine Großbank in Form der Aktiengesellschaft. Entscheidungsleitend sollte dabei aber immer sein, die gewünschten Wertevorstellungen des Unternehmens abzugleichen mit den Werten, die durch das jeweilige Modell der Führungsethiken in die Praxis transferiert werden. Ohne einen behutsamen Abgleich mit der Wertebasis des Unternehmens holt man sich womöglich zunächst unbemerkt mit der Implementierung eines schön klingenden Konzeptes 
der Führungsethik ein trojanisches Pferd ins Unternehmen. Dann ändern sich nach und nach Regeln und Verhaltensweisen entsprechend dieses Konzeptes, es wächst eine moralische Kultur heran, die möglicherweise den gewünschten Grundwerten des Unternehmens widerspricht. Und am Ende sind dann die Geister schwer zu bändigen, die man selber rief. Um das zu verhindern, gilt es, das führungsethische Paradigma, das die Unternehmenskultur festigen, weiterentwickeln oder verändern soll, achtsam auszuwählen. Der vorliegende Beitrag will dafür eine praktische Entscheidungshilfe sein.

Was also macht überhaupt eine Führungstheorie zur Führungsethik? Die Antwort ist keineswegs trivial: Zahlreiche Leitfäden für die Praxis fokussieren vor allem Tipps zum guten Führungsverhalten in den vielfältigen internen und externen Unternehmensbeziehungen, etwa in Konflikten oder Veränderungs-Prozessen. Viele Prinzipien wurden hierfür schon entworfen und an konkreten Beispielen anschaulich illustriert. Und auch Tugendkataloge für Manager und Führungskräfte haben Konjunktur. Hier finden sich dann zahlreiche individuelle Eigenschaften, die Erfolg versprechen in Motivation, Kommunikation, Vertrauensbildung etc. Das alles kann hilfreich sein, in konkreten Dilemma-Situationen Orientierung zu finden, oder auch erfolgreich erprobte Instrumente und Tricks kennenzulernen. Brauchbares Handwerkszeug eines erfolgreichen Managements kommt direkt zum Punkt, ist praxisnah und anwendungsorientiert. Eine stimmige Wertekultur guter Führung zu entwerfen, ist dann naturgemäß nicht das erste Ziel. Dabei transportieren die allzu oft neutral daherkommenden Handwerkskoffer schleichend selbst oft ein bestimmtes Bild vom Menschen und von der Gestaltung zwischenmenschlicher Beziehungen oder eine mehr oder minder ausgeprägte Wertschätzung der Schöpfung, ohne diese Inhalte immer offenzulegen. Ebenso stellen sie es zwangsläufig in ein normatives Verhältnis zur Wirtschaftlichkeit.

\section{Ein Beispiel}

Was oft zu lesen ist: Mitarbeiter gelten als die wichtigste Ressource der Unternehmen. ${ }^{1}$ Diese zu optimieren, ist Ziel des Human Ressource Managements. Es handelt sich aber um ein doppeldeutiges Ziel: Einerseits kann es bedeuten, den Menschen gegenüber anderen Ressourcen als besonders wertvoll herauszuheben und in der Personalführung vor allem anderen die humane Entfaltung als Personen anzustreben. Dann kann von einer Humanorientierung des Unternehmens als Führungsethik gesprochen werden. Andererseits kann es heißen, dass der Mensch als Mitarbeiter eines

1 Vgl. o.V.: Handelsblatt (2005); Rodeck (2012).
Unternehmens einfach nur eingereiht wird in ein Kontinuum des Ressourcenpools, den es unter Marktbedingungen insgesamt zu optimieren gilt. Dann geht es in der Führung zuerst nicht um den Menschen und seine Entfaltung, sondern im Sinne kalkulierender ökonomischer Ethik zuerst um eine effiziente Allokation der knappen Ressourcen, unter denen der Mensch gerade als besonders wichtig angesehen ist. Ist der einzelne Mitarbeiter zuerst ein austauschbarer Produktionsfaktor, dann geht es im Unternehmen um die summierte Optimierung der Humanressourcen in Abhängigkeit des durch Anreize zu optimierenden Produkts aus Motivation und Qualifikation der Mitarbeiterschaft. Weitere Präferenzen und Persönlichkeitsmerkmale der Mitarbeiter (Gefühle, Werthaltungen, Vorlieben) könnten dann vernachlässigt werden. Wird dagegen der Mensch zuerst als Selbstzweck verstanden, dann geht es im Miteinander der Menschen im Unternehmen nicht allein um die Optimierung einer summierten Ausbringungsmenge, sondern auch um die personale Entwicklung jedes einzelnen Menschen auch jenseits seines Beitrags zur OutputSteigerung. Dann steht die Entfaltung jedes Einzelnen im Mittelpunkt und nicht allein eine Summe, in der der eine oder andere unter die Räder kommen kann. Entweder verbirgt sich hinter dem Bekenntnis zum Menschen als wichtigster Ressource also die humane Entfaltung der Mitarbeiter oder ökonomisches Effizienzkalkül als das leitende Motiv. Das bloße Bekenntnis zum Menschen als Mittelpunkt der Führung reicht nicht aus, um deren ethischen Gehalt einordnen und bewerten zu können.

Solche ethischen Hintergründe der jeweiligen Wertbasis von Führungskonzepten mit ihrem Menschenbild sind - das sei zuzugeben - für die konkreten Entscheidungssituationen und selbst für Leitbild- oder ChangeDiskussionen und Umsetzungen im Unternehmen zunächst wohl von nachrangiger Bedeutung. Dennoch bestimmen gerade solche normativen Voraussetzungen - meist hinter all den guten Ratschlägen versteckt unbemerkt einen postulierten Geist guter Führung. Bevor solche Konzepte im Unternehmen zur Anwendung kommen, sollte die Wertebasis der Führungskonzepte, -modelle, -theorien - offengelegt werden, gerade in einem Unternehmen, dass ausdrücklich eine $\mathrm{zu}$ ihr passende Kultur der Führungsethik implementieren will. Das gekonnte Manu agere des Managements allein ist im moralischen Sinn noch keine gute Führung. Denn gut ist keine deskriptive Kategorie für eine bloß richtige und vielleicht sogar erfolgreiche Gestaltung unter Anwendung vorgeschlagener Instrumente. Führungsethik geht weiter und fragt ausdrücklich nach Inhalt und Begründung der Wertebasis, die, wenn sie glaubwürdig sein will, nicht einfach auf Lippenbekenntnisse in Hochglanzbroschüren reduzierbar ist. Sie kann etwa religiös motiviert sein oder 
politisch, anthroposophisch, aufgeklärt-humanistisch o.a. weltanschaulich inspiriert. Stimmen Unternehmenswerte und gelebte Werte in der Unternehmenskultur überein, dann ist die passende Führungsethik als Führungsmoral implementiert. Glaubwürdigkeit, Vertrauen und Transparenz machen es nötig, dass die Unternehmenswerte in Leitbildern und die praktische Führungsmoral möglichst die gleichen Werte gleich gewichten. Und im Markt kann eine solche Kohärenz ein nicht zu unterschätzender Wettbewerbsvorteil sein, sowohl nach innen für die Identifikation der eigenen Mitarbeiter wie auch nach außen als Marketingsignal.

\subsection{Darum Führungsethik Sozialer Marktwirtschaft}

Eine systematische Identifizierung von Führungsethik in Abgrenzung zu weiteren Führungstheorien wäre die Aufgabe für einen Lexikonartikel. Hier soll es darüber hinaus um die Frage gehen, welche Führungsethik von ihrer Grundidee her mit den normativen Grundideen Sozialer Marktwirtschaft übereinstimmt. Diese gelten hier im Rahmen einer Makro-Mikro-Perspektive als ein postulierter Referenzrahmen für eine legitime Unternehmens- und Führungsethik. Damit ist nicht etwa eine einfache Übertragbarkeit der voraussetzungsreichen Wirtschaftsordnungskonzeption auf den Bereich Führung behauptet. Vielmehr gehe ich davon aus, dass (1.) die Soziale Marktwirtschaft von der Grundidee her eine legitime und anzustrebende Wirtschaftsordnung ist, (2.) dass sie auf der Makroebene eine Ordnungsethik mit Grundwerten und ethische Prinzipien für ein gesellschaftliches Kulturprogramm anbietet, und (3.) dass diese Werte und Prinzipien der Ordnungsethik ein Vorbild sein können für eine gute Unternehmenskultur. Weil also sich Grundwerte und ethische Prinzipien auf der Makroebene bewährt haben, werden diese nun für die Führungsethik kultiviert. Führungsethiken Sozialer Marktwirtschaft übertragen die ethische Kulturidee der Ordnung auf das Unternehmen und dienen zugleich einer inneren Stringenz und Stabilität seiner normativen Grundidee.

Der hier gewählteAnstoß geht von der Ebene ethischer Führungskultur aus, da diese wie auf der Makroebene die ordnungspolitische Idee ein Kulturprogramm sozialen Zusammenhalts vorstellt. Führung ist dabei nicht allein als Aufgabe des Personalmanagements zu verstehen, sondern in einem weiteren Sinn als „intendierte Verhaltensbeeinflussung" (von Oelsnitz 2012, S. 8). Als Gegenstand der Ethik ist sie in allen Unternehmensbereichen, Abteilungen und Hierarchieebenen "die verantwortbare Beeinflussung des Verhaltens der Geführten“ (Kuhn und Weibler 2012, S. 15). Führungskultur ist "a composite of people's behaviors within the organization and in the underlying shared beliefs, meaning and values, the norms commonly applied and the practices carried out" (Melé und Cantón 2014, S. 45). Sie ist kontextuell in die gesellschaftliche Kultur eingebunden und prägt sie im Sinne des Stilgedankens normativ mit. Dafür sollen Kriterien einer Führungsethik bestimmt werden, die zugleich der normativen Grundidee Sozialer Marktwirtschaft entspricht. Solche Konzepte sollen in Unternehmen zur Anwendung kommen. Für die Unternehmen gibt es damit eine Orientierung im Dschungel. Damit kann es gelingen, durch entsprechend identifizierte Führungsethik und -kultur das Profil einer Kultur Sozialer Marktwirtschaft in Deutschland theoretisch neu zu schärfen und praktisch neu zu beleben. Ein solcher Identifizierungs- und Kultivierungsansatz ist keineswegs trivial. Bei den ethisch daher kommenden Konzepten der Führung dürfen die (etwa normativ individualistisch, deontologisch oder metaphysisch) normativen Grundlagen nicht hinter dem anschaulich präsentierten Handwerkzeug effektiven Managements zurücktreten, so dass der Ethik-Begriff dann selbst zur Leerformel wird. Der vorliegende deduktive Beitrag will deshalb als Modell einen Leitfaden anbieten, um damit aus ordoliberaler Perspektive Sozialer Marktwirtschaft Führungsethik von Führungstheorie zu unterscheiden und die Vereinbarkeit von angebotenen Ethikansätzen anhand der relevanten ethischen Perspektiven mit transparenten normativen Prinzipien zu identifizieren. Damit können Ansätze der Führungsethik auch entsprechenden Schulen zugordnet werden, die sich zurecht bzw. zu Unrecht auf die Soziale Marktwirtschaft berufen. Hierzu wird als Referenz keine Ordnungstheorie rekonstruiert oder gar neu entworfen. Vielmehr werden für eine Führungsethik Sozialer Marktwirtschaft die für diesen Bereich relevante Wertebasis und daraus folgende Prinzipien identifiziert zur Anwendung gebracht.

Die grundsätzliche Identifizierung von Führungsethik im Gegensatz zur Führungstheorie ist das erste, die systematische Offenlegung von alternativen führungsethischen Wertebasen mit ihren Konsequenzen für die gelebte Kultur das zweite, das Plädoyer für eine an den ethischen Grundwerten unserer wirtschaftspolitischen Kulturidee orientierten Führungskultur das dritte Anliegen dieses Beitrages.

\section{Ein Leitfaden}

\subsection{Ethikbegriff}

Für unsere Diskussion muss der zugrundeliegende Ethikbegriff näher geklärt werden, der als Systematik von der gelebten Führungsmoral zu unterscheiden ist und 
dabei theologische und nicht theologische Wertesystematik umschließt: Führungsethik im Allgemeinen ist eine Führungstheorie, die explizit mit einer transparenten Wertesystematik Führungspraxis normativ bewertet. Sie entwirft dazu theoretisch eine normative Systematik mit transparenten Werten. Die Werte sind dann selbst die Grundlage dazu, Führungspraxis normativ bewerten zu können. So lässt sich die Bedeutung von Normativität in der Führungsethik in Abgrenzung zu den Praxisfeldern Führung und Management so illustrieren:

\section{Beispiel}

Nun könnte man sagen, die Frage nach einer moralisch guten Führung entscheide sich allein an ihrer ökonomisch erfolgreichen Wirksamkeit. Gerade im Kontext Marktwirtschaft sei es schließlich das angestrebte Ziel, durch solchen Erfolg den Erhalt oder das Wachstum des Unternehmens zu sichern und Arbeitsplätze zu schaffen. Dies ist zweifellos eine wirtschaftsethisch positiv zu bewertende Konsequenz von Führung. Der Hinweis auf ökonomisch o.a. erfolgreiche Konsequenzen ist aber nicht hinreichend, weil nicht nur die (in monetärem o.a. Ertrag messbaren) Konsequenzen, sondern die gelebte Führungskultur selbst Gegenstand dieser Ethik sein muss.

Es wird in einem nächsten Schritt das Wesen von Führungsethik weiter konkretisiert, nunmehr vom allgemeinen zu einem speziellen Verständnis einer Führungsethik im engeren Sinne.

Eine Führungsethik im engeren Sinne muss auf der Grundlage einer transparenten Wertebasis und auf ihren Gegenstandsbereich bezogen für ihre normative Bewertung der Führungskultur das Verhältnis von Wirtschaftlichkeit und Menschendienlichkeit bestimmen,

\begin{tabular}{|c|c|c|c|}
\hline & Management & Führung & Führungsethik \\
\hline Aufgabe & $\begin{array}{l}\text { Praktische } \\
\text { Orientierung } \\
\text { geben als erlerntes } \\
\text { Handwerk }\end{array}$ & $\begin{array}{l}\text { Praktische } \\
\text { Orientierung } \\
\text { geben auch } \\
\text { mit Werten }\end{array}$ & $\begin{array}{l}\text { Normative } \\
\text { Bewertung von } \\
\text { Führung }\end{array}$ \\
\hline Bewertung & $\begin{array}{l}\text { richtig oder falsch } \\
\text { im Sinne der } \\
\text { Effektivität }\end{array}$ & $\begin{array}{l}\text { effektiv und } \\
\text { moralisch } \\
\text { gut oder } \\
\text { schlecht im } \\
\text { Sinne der } \\
\text { Werte }\end{array}$ & $\begin{array}{l}\text { ethisch gut } \\
\text { oder schlecht: } \\
\text { Hinterfragung } \\
\text { der gelebten } \\
\text { Werte } \\
\text { mithilfe einer } \\
\text { transparenten } \\
\text { normativen } \\
\text { Systematik }\end{array}$ \\
\hline Ziel & Wirksamkeit & $\begin{array}{l}\text { Wirksamkeit } \\
\text { und } \\
\text { praktische } \\
\text { normative } \\
\text { Kohärenz }\end{array}$ & $\begin{array}{l}\text { theoretische } \\
\text { normative } \\
\text { Kohärenz }\end{array}$ \\
\hline
\end{tabular}

Tabelle 2.1 Management - Führung - Führungsethik Quelle: Eigene Darstellung ohne eines der Ziele zu instrumentalisieren oder zu substituieren. $^{2}$

Oder anders: Führungsethik im engeren Sinne muss für den Bereich Führung normativ bewerten, wie Wirtschaftlichkeit und Humanorientierung dort als eigenständige Selbstzwecke zu verstehen und aufeinander bezogen werden sollen. Ein solches, enges Verständnis ist hier zugrunde gelegt.

\subsection{Grundfrage der Wertebasis}

Der im Folgenden entwickelte Leitfaden ist eine Orientierungshilfe zur Identifizierung von Führungsethiken, die mit den Grundideen Sozialer Marktwirtschaft vereinbar sind. Daraus folgt, dass kollektivistisch oder frei marktwirtschaftlich konzipierte Ethiken diesem Kriterium nicht entsprechen.

Die Identifizierung der Semantik einer Kultur Sozialer Marktwirtschaft beginnt im ursprünglichen ordoliberalen Sinne - und daran sei hier angeknüpft - mit einem grundsätzlichen Bekenntnis zum Markt. Sie ist eine Konkurrenzwirtschaft mit den ethisch wünschenswerten effizienten Allokationen, durch die Ressourcenverschwendung vermieden wird (Goldschmidt 2006). Führungsethik im Sinne Sozialer Marktwirtschaft muss deshalb das marktwirtschaftliche Ziel ökonomischer Effizienz verfolgen. Dieses Ziel ist notwendig, aber nicht hinreichend. Auf der Ebene der Wirtschaftsordnung wird ein Rahmen gefordert, der begründungspflichtige Redistributionen und andere Marktregulierungen gestattet. Die Gründerväter Sozialer Marktwirtschaft kommen dabei zu je eigenen inhaltlichen Auslegungen dieser den Markt und seine Eigengesetzlichkeiten rahmenden Prinzipien. Grundlegend ist stets ein als universalisierbar begründetes Humanziel. Dieser starke Anspruch ist bei Eucken kantisch, bei Müller-Armack u.a. religiös begründet (Müller und Nass 2013). So fordert etwa Eucken (1952/2004, S. 179) in Anlehnung an Kants Kategorischen Imperativ in der dritten Fassung eine „Wirtschaftsordnung ..., in der die Menschen nicht nur Mittel zum Zweck, nicht nur Teilchen des Apparates sind“. Der Sozialismus wird von inm vor allem deshalb abgelehnt, weil durch die angestrebte Vereinigung

2 Vgl. die analoge Unterscheidung von Human- und Erfolgsverantwortung der Fürhung bei Kreuer (2017), S. 5-6, 18 sowie vergleichbare Vorstellungen eines solchen für Führungsethik konstitutiven Zieledualismus bei Kuhn und Weibler (2012), S. 46 oder Ulrich (1999), S. 230, 237. Für die Personalentwicklung definiert es Becker (2002), S. 492 im Effizienzmodus so: „Personalentwicklung dient der Erreichung der Unternehmensziele (wirtschaftliche Effizienz) und die Verwirklichung individueller Entwicklungsziele der Mitarbeiter (soziale Effizienz). "Vgl. auch Ulrich (2010), S. 28, Kuhn und Weibler (2012a), S. 23, 94, 107; Fischer und Fischer (2007), S. 22. 
wirtschaftlicher Konzentration der Mensch zur Sache wird und seinen Charakter als Person verliert: „Der Apparat ist Zweck, der Mensch Mittel“ (Eucken 1952/2004, S. 177). Soziale Marktwirtschaft steht unbedingt im Dienst an der Entfaltung des Menschen als Person. Das von Eucken transzendental begründete Humanziel der Personalität ist auf Ebene der Führungskultur bindend, will diese mit der Grundidee der Wirtschaftsordnung konvergieren. Es betont kategorisch die Selbstzwecklichkeit des Menschen und seine Entfaltung als Person. Es ist der Grund für die Rahmung des Marktes und wirft in der Führung die Frage auf, welche Rolle ihr gegenüber dem Effizienzziel zukommt. Diese normative Bipolarität muss eine Führungsethik abbilden. Ethisch orientiertes Personalmanagement hat das Leistungsziel, das Unternehmen mit guten Mitarbeitern zu versorgen. Es hat zugleich das Humanziel, die Mitarbeiter durch das Unternehmen zu versorgen (Plaschke et al. 2007). Im Sinne der Gründerväter Sozialer Marktwirtschaft lässt sich auch auf dieser Ebene über die Gewichtung der Ziele streiten. Im Sinne der weiten Definition von Führung ist diese Bipolarität der Ziele aber als unbedingte Wertebasis konstitutiv für eine Führungsethik Sozialer Marktwirtschaft. Ihre Ausgestaltung muss systematisch den führungsrelevanten Verantwortungsdualismus von Effizienz- und Humanziel auslegen.

Während das Effizienzziel durch das ökonomische Marktprinzip inhaltlich vorgegeben ist, ist die Semantik des Humanziels umstritten. In der Wirtschaftsethik finden sich dazu miteinander konkurrierende Paradigmen: Entweder wird im Sinne etwa von K. Homann eine monologische Heuristik des Homo Oeconomicus als Orientierung des Humanziels angenommen. Oder es wird auf anthropologischer Grundlage eine komplexe dialogische Rationalität des Menschen angenommen, wiesieSmith, Ulrich, Senu.a. vertreten. Dann sind jeweils egoistische und komplexe ethische Motive des Menschen in der Humanorientierung zu berücksichtigen. ${ }^{3}$ Die Wertebasis verlangt von einer Führungsethik Sozialer Marktwirtschaft somit eine Entscheidung über das Grundverständnis der jeweiligen Humanorientierung. Es sind als Paradigmen eine monologische Heuristik von einer dialogischen Anthropologie zu unterscheiden.

\subsection{Drei Kulturprinzipien der Führung}

Die Kulturprinzipien dienen einer Beantwortung derFrage nach dem Verhältnis von Effizienz- und Humanziel. Sie

3 Eine normative monologische Heuristik jenseits der egoistischen Rationalität wäre etwas im Sinne des linken Libertarianismus von Philippe Van Parijs denkbar, wurde unternehmensethisch bislang nicht vorgelegt und ist zudem unvereinbar mit einer marktwirtschaftlichen Logik. sind im Sinne der Wertebasis kategorisch zu verstehen. D.h. sie sind nicht in einem kreativen Schöpfungsprozess immer wieder neu zu konstruieren oder konsensual auszuhandeln. Vielmehr werden sie verstanden als Prämissen, die die Ethik nicht selbst aus sich hervorbringt. Erweiterungen des Katalogs aber sind damit nicht ausgeschlossen. Transzendentale und religiöse Begründungen für die Prinzipien entsprechen der Grundidee. Daran anschlussfähige alternative Begründungen finden sich in der neu-aristotelischen Befähigungsidee (z.B. Sen) bzw. in einem neumetaphysischen Ansatz, wie inn Schramm (2015) vertritt. Danach liegen in Anlehnung an Hartshorne (1948/1964) und Whitehead (1929/1978) in einer angenommenen sozialen Struktur des Universums als moralischer Wahrheit „unbeliebige Vorgaben einer realitätsangemessenen Ethik" vor, die so eine „kosmologische Werthaltigkeit“ (Schramm 2015, S. 179) quasi transzendental voraussetzen. Verschiedene Zugänge erlauben also die Begründung für objektiv gehaltener Prinzipien. Zur Lösung des Dualismus von Human- und Effizienzziel sind als solche Solidarität, Subsidiarität und Marktkonformität unbestritten. Eucken ergänzt als universalisierbare regulative Prinzipien den Vorrang der Währungspolitik, die Konstanz der Wirtschaftspolitik, offene Märkte, Vertragsfreiheit, Haftung, Privateigentum, Monopolkontrolle, progressive Besteuerung, die Vermeidung externer Effekte und Interventionen gegen Anomalien auf dem Arbeitsmarkt (Goldschmidt 2006). Des Weiteren ist der irenische Stilgedanke gesellschaftlichen Zusammenhalts ein wesentliches Kulturprinzip.

Im Sinne semantischer Klarheit und Komplexitätsreduzierung werden die Prinzipien nun zu einem Katalog mit führungsethischer Relevanz transformiert.

1. Der irenische Stilgedanke ist eine kulturelle Integrationsformel, die eine Symbiose aus Wirtschafts-, Sozial- und Kulturpolitik unterstellt. Sie zielt auf die Sicherung des sozialen Friedens und beschränkt sich nicht auf anonymen Regelgehorsam. In seiner Interpretation zu Rüstow beschreibt Hegner (2000, S. 38) dafür die Gefährdung sozialen Friedens so: „Der Verlust übergeordneter Werte, die für den Zusammenhalt von Solidargemeinschaften notwendig sind, und die dem einzelnen eine Orientierung in Bezug auf sein Handeln geben, begünstigt die Verrohung des einzelnen sowie die gegenseitige Entfremdung“. Die Ausbildung gegenseitigen Vertrauens der Starken gegenüber den Schwachen und umgekehrt dient der politischen Implementierbarkeit Sozialer Marktwirtschaft (Ebd., S. 90). Mit dem Prinzip gegenseitigen Vertrauens von Führungskräften und Mitarbeitern 
kann die Führungsethik gefragt werden: Wie wird Vertrauenskultur begründet, verstanden und im implementiert?

2. Subsidiarität und Marktkonformität begründen den Leistungsgrundsatz, nach dem sich Stellung, Entlohnung und übertragene Verantwortung richten sollen. Leistungs- und Haftungsgrundsatz gemeinsam mit der Vermeidung externer Effekte stehen für die Eigenverantwortung. Solidarität, progressive Besteuerung und auch die verfassungsgemäße Sozialpflichtigkeit des Eigentums stehen für die Sozialverantwortung. Mit dem bipolaren Prinzip der Verantwortung kann dann die Führungsethik gefragt werden: Wie werden Eigenund Sozialverantwortung begründet, verstanden, gewichtet und implementiert?

3. Das Doppelprinzip der Verantwortung kann inhaltlich angereichert werden durch ein Freiheitsprinzip. Vertragsfreiheit und Schutz des Privateigentums stehen nicht nur für eine Ablehnung des Kollektivismus, sondern auch für die Vermeidung von Zwang, einem im Sinne von Hayeks Grundübel freiheitlicher Ordnung (vgl. grundsätzlich Battyány 2007). Sie begründen eine Forderung negativer Freiheit als Abwehrrecht. Dies reicht aber nicht aus. Es kommt mit der Sozialverantwortung die Forderung positiver Freiheit hinzu. Sie findet sich im neu-aristotelischen Befähigungsansatz von Sen konkretisiert. Dieser postuliert vorpositiv begründete menschliche Grundrechte auf die Entfaltung von grundlegenden Fähigkeiten wie Gesundheit, Kreativität, Eigenverantwortung oder soziale Integration (Sen 1993, S. 31). Sie sind Ausdruck der Freiheit und stehen jedem Menschen zu. Die Selbstbestimmung ist als ein Entscheidungsraum konzipiert, der jedem Menschen objektiv zusteht. Freiheit ist danach ein individueller Optionsraum verantwortlichen Entscheidens, der angemessene Wahlmöglichkeiten zwischen Alternativen erlaubt. Gerechtigkeit fordert, dass die Individuen zu einer solchen positiven Befähigungsfreiheit zu befreien sind, indem Hindernisse der Entscheidungsfreiheit beseitigt werden. Egalisiert werden soll dabei nicht die tatsächlich erhaltene Ausstattung mit Grundfunktionen, sondern die Befähigung, solche Grundfunktionen entfalten zu können. Soziale Rechte verpflichten auf Makroebene den Staat darauf, die Wahlmöglichkeiten zur Entfaltung der menschlichen Grundfunktionen etwa mit entsprechenden Bildungsund Gesundheitseinrichtungen bereitzustellen (Sen 2003, S. 36). Die Wahlmöglichkeiten optimieren aber erst dann den Verantwortungsraum, wenn die Individuen selbst in der Lage sind, eigenverantwortlich entscheiden zu können (Nussbaum 1988, S. 115). Wie der Einzelne die inm angebotene Bildung annimmt, ob der Kranke die inm offenstehenden Krankenhäuser nutzt, das liegt in der Verantwortung der Individuen. Als Suffizienz ist neben der Sicherung eines überlebenssichernden Mindestbedarfs die Schaffung eines solchen Verantwortungsraumes positiver Freiheit zu verstehen, die den Individuen die innen mögliche Entwicklung ihrer Talente ermöglicht, ihnen bei selbst verursachter Auslassung dieser Chancen aber keine unbedingten Rechte auf einen kompensierenden Ausgleich zuspricht. Dies entspricht dem Haftungsgedanken. So wird im Sinne Sozialer Marktwirtschaft der Verantwortungsgedanken interpretiert. Mit inm muss die Führungsethik gefragt werden: Entspricht das vertretene Verantwortungsverständnis einer solchen Kultur der Befähigungsfreiheit?

Die hier aufgestellte Behauptung, die Befähigungsfreiheit repräsentiere die normative Grundidee Sozialer Marktwirtschaft, ist nicht evident. Denn dieses Prinzip entspringt der Logik der dialogischen Anthropologie, die auch Sen vertritt. Die Forderung einer Entfaltung menschlicher Grundfunktionen interpretiert das semantisch umstrittene Humanziel der Wertebasis und damit auch die Grundidee Sozialer Marktwirtschaft im Sinne der Befähigungsfreiheit. Dieses Prinzip kann deshalb kein Kriterium sein für die Bewertung einer Führungsethik im Paradigma monologischer Heuristik, sondern allein in dem Kontext, dem es entnommen ist. Wenn hier dieses Prinzip eingeführt wurde, so deshalb, weil es von seiner Begründung und Semantik m.E. kongruent zu den Prinzipien der Gründerväter erscheint. Ein Verständnis Sozialer Marktwirtschaft, die das Humanziel als monologische Heuristik deutet, wird an diese Stelle ein anderes Prinzip setzen, das ihrem Paradigma entspricht. Wenn also im Folgenden der Leitfaden weiterentwickelt und später zur Anwendung gebracht wird, so ist zu berücksichtigen, dass dieser allein das anthropologische Paradigma vertreten kann.

\subsection{Drei Perspektiven ethischer Bewertung}

Für die Bewertung anhand der Kulturprinzipien sind die Führungsethiken relevanten ethischen Perspektiven auszusetzen. Zu fragen ist nach gerechten Beziehungen im Unternehmen immer mit Blick auf Akteure und Regeln. Daraus ergeben sich unmittelbar zunächst zwei Perspektiven. Die Führungskultur ist realisiert im Ethos der Beteiligten: der Führungskräfte und der Mitarbeiter. Deshalb ist Führungsethik zunächst 


\section{Kategorische Wertebasis}

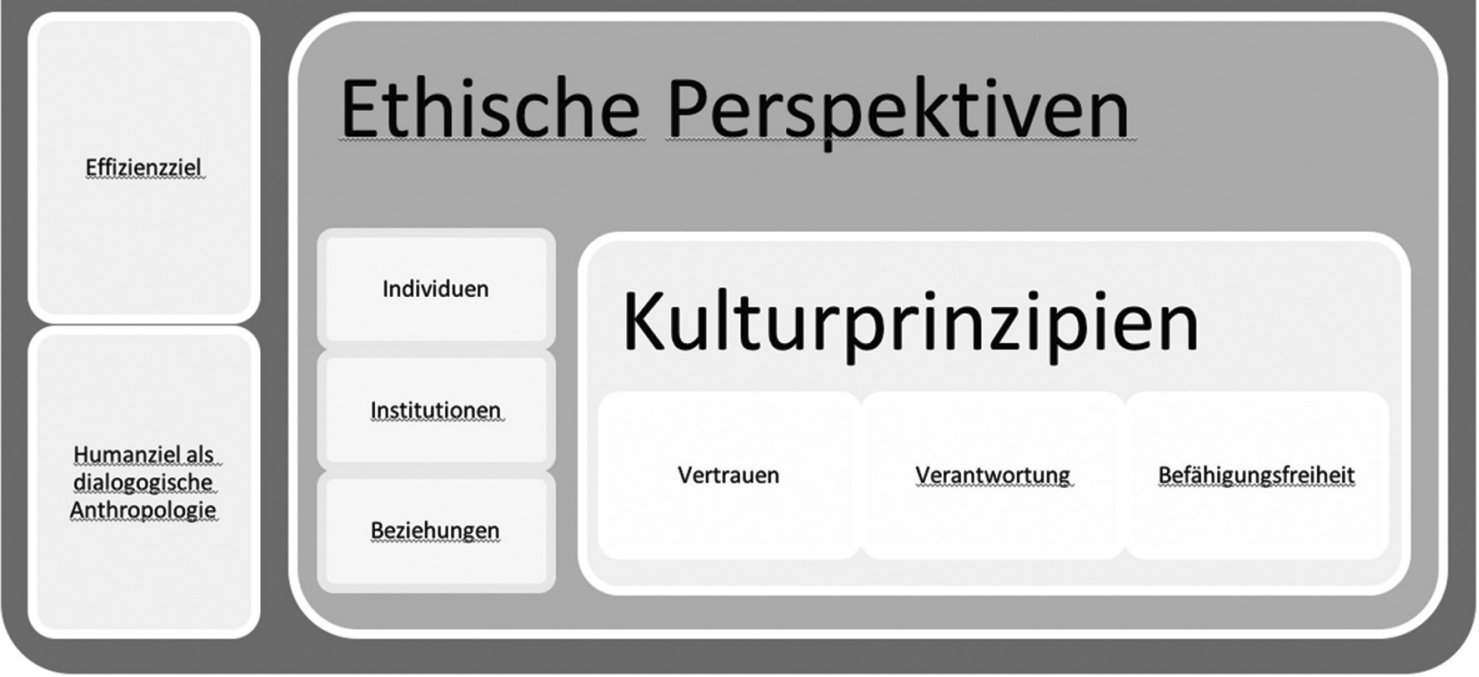

Abbildung 2.1 LIFESMA - das Modell

Quelle: eigene Darstellung.

Individualethik, erschöpft sich aber nicht darin. ${ }^{4}$ Sie ist auch Institutionenethik und fragt Personal-, Organisations- oder Grundsatzabteilungen etwa nach der Gestaltung der geschriebenen und ungeschriebenen Unternehmensregeln wie nach Leitbildern, Organigrammen, Vorgaben für Hierarchie, Entscheidungswege und Controlling wie für die Zuteilung von Kompetenzen und Delegation. Ebenso fragt sie nach den Strategien der angestrebten Kommunikations- und Motivationskultur sowie von Personalplanung, -einsatz und -entwicklung. Daraus ergeben sich konkrete Antworten etwa auf folgende Fragen: Welche Charaktere sollen sich als Führungskräfte durchsetzen? ${ }^{5}$ Welche Mitarbeiter sollen eingestellt werden? Welche Bildungsmaßnahmen sind zu fördern? Sollen Führungskräfte in ihrem Verantwortungsbereich mehr auf kooperative oder auf Top-down-Entscheidungen, mehr auf Kontrolle oder auf Vertrauen, mehr auf Konkurrenz

4 Dagegen wendet Rohrhirsch (2013, S. 181 ff.) aus christlicher Sicht ein, es gebe keine christliche Führungsethik, sondern nur christliche Führungskräfte. Einer solchen Fokussierung auf die Tugendethik wird hier nicht gefolgt. Ihre Zurückdrängung wird im heuristischen Paradigma favorisiert. Homann (1993, S. 41) warnt vor einer nicht ökono-misch endogenisierten Moral der Führungskräfte und Mitarbeiter. Diese Sicht für einen entsprechenden Leitfaden weiter zu verfolgen und zu systematisieren, ist wiederum Aufgabe der Vertreter dieses Paradigmas

5 Kuhn und Weibler (2012, S. 93 f.) kritisieren etwa, dass derzeit vor allem Machiavellisten. Narzissten oder Psychopa-then das Rennen machen. oder Team setzen? Sind hier vor allem extrinsische finanzielle Anreize oder intrinsische Motivation durch Einsicht und Identifikation zu favorisieren?

Eine dritte Perspektive stellt das wechselseitige Verhältnis der ersten beiden als einen eigenen Blickwinkel heraus. Führungsethik ist in diesem Sinne Beziehungsethik, die sich als eingeforderte Kultur im konkreten Miteinander von Führungskräften und Mitarbeitern realisiert. Dazu zählen deren Verhalten zu den Regeln und Erwartungen und die Rückwirkungen auf die institutionelle Ebene. Diese dritte Perspektive berücksichtigt, dass Institutionen in den Transaktionen konkret werden, die im Sinne des dynamischen Stilgedankens fortwährend auf die Führungskultur als ganze (auf Individualethos der Beteiligten und Institutionen) evolvierend einwirken, was wiederum die Beziehungen verändert (Schramm 2015, S. 184 f.). Diese fortwährend reziproken Verhältnisse bilden eigene Entitäten gelebter Kultur. Sie sind geprägt von Inhalt und Grad der Loyalität bzw. Identifikation der Führungskräfte und Mitarbeiter zum Unternehmen mit seinen Regeln und Erwartungen.

\subsection{LIFESMA - das Modell}

Der normative Leitfaden kann nun in ein einfaches Modell überführt werden. Dazu erfolgt eine Einteilung in Prinzipien, Perspektiven und Bewertung. Grundfrage und Prinzipien gelten als kategorisch. Die einzelnen Bewertungen erheben dagegen keinen kategorischen 
Anspruch. Sie sind wissenschaftliche Interpretationen, die unterschiedlich ausfallen können. Das dreidimensionale Modell ist selbst keine Norm, sondern als Bewertungsinstrument eine komplexitätsreduzierende Orientierung für die Identifizierung einer Führungsethik Sozialer Marktwirtschaft. Der „Leitfaden zur Identifizierung von Führungsethik Sozialer Marktwirtschaft" wird im Folgenden „LIFESMA“ abgekürzt.

Wird eine Theorie der LIFESMA-Prüfung unterzogen, so ist im ersten Schritt zu entscheiden, ob es sich um eine Ethik handelt: Sie muss dazu die Grundfrage der Wertebasis zum Thema haben. In einem zweiten Schritt ist die Zuordnung zu einem der beiden Verständnisse des Humanziels vorzunehmen.

Dann sind in einem dritten Schritt aus Sicht der drei Perspektiven die drei Prinzipien zu prüfen, so dass hier neun Bewertungen vorzunehmen sind. Damit kann tabellarisch eine Übersicht über die schwach und stark ausgeprägten Kulturprinzipien im Kontext des gewählten Paradigmas zum Humanziel aufgestellt werden, wobei mit dem Prinzip der Befähigungsfreiheit im eben genannten Sinne das anthropologische Paradigma weiterverfolgt wird. Ergebnis der Bewertung ist dann in einem vierten Schritt die Einstufung eines Modells etwa als eine starke oder schwache Theorie Sozialer Marktwirtschaft. Die Gewichtung der neun Bewertungsfelder untereinander ist mit dem Leitfaden noch nicht geleistet. Sie bleibt Gegenstand der wissenschaftlichen Interpretation.

\section{Anwendung mithilfe der Wertebasis}

Es können nun die vier Schritte vorgestellt werden, mit denen LIFESMA bei der Bewertung von Führungstheorien zur Anwendung kommt.

\subsection{Erster Schritt: Führungsethik oder -theorie?}

Erster Schritt der Anwendung ist die Identifizierung einer Führungsethik Sozialer Marktwirtschaft mithilfe der Wertebasis. Notwendig eingefordert wird unserem grundlegenden Verständnis von Führungsethik entsprechend ein Miteinander von Effizienz- und Humanziel, wobei keines durch das andere aufgehoben werden darf. Es wird damit vorausgesetzt, dass keiner der Werte dem anderen ganz geopfert wird. Gerät in einer Theorie eines der beiden Ziele aus dem Blick, führt ihre Umsetzung in der Unternehmenskultur zumindest langfristig entweder zu einer ökonomistischen Depersonalisierung oder zu einer utopischen Entwirtschaftlichung unternehmerischen Handelns. Wirtschaftlichkeit und Menschendienlichkeit, Effizienz und Humanorientierung müssen in einer Theorie also jeweils beide als Ziele von Führung anerkannt sein, um dabei von einer Führungsethik sprechen zu können. Daraus folgt, was keine Führungsethik in diesem Sinne ist: Theorien, die das Wirtschaftlichkeitsziel als Ziel der Führungskultur ausschließen oder außer Acht lassen, können möglicherweise für andere Lebensbereiche interessant sein, für die Unternehmensführung in der Marktwirtschaft sind sie ungeeignet. Theorien, die die Menschendienlichkeit als Ziel für die Führungskultur ausschließen oder außer Acht lassen, können möglicherweise für Wirksamkeitsstudien interessant sein, als Ethik sind sie dann aber nicht geeignet. Führungsmodelle, die den Humanaspekt zugunsten einer bloßen Selbstzwecklichkeit der Effizienz ausblenden, fallen dann nicht unter die Ethik. Paradigmen der Führungsethik stellen also theoretische Modelle vor, die für den Bereich Führung aufzeigen, wie Wirtschaftlichkeit und Humanorientierung als Selbstzwecke zu verstehen und aufeinander bezogen werden sollen. Notwendig eingefordert für Führungsethik wird also ein Miteinander von Effizienz- und Humanziel, wobei keines durch das andere aufgehoben werden darf. Dieser Verantwortungsdualismus fokussiert in der Führungsethik eine moralische Sicht auf jeden Mitarbeiter (vgl. etwa Fischer und Fischer 2007, S. 22).

Eine Führungstheorie jenseits der Ethik kann also durchaus so argumentieren: Ist der Mitarbeiter aus Sicht der Führungskraft zuerst ein austauschbarer Produktionsfaktor, dann geht es in der Führung um die summierte Optimierung der Humanressourcen im Unternehmen in Abhängigkeit des durch Anreize zu optimierenden Produkts aus Motivation und Qualifikation der Mitarbeiterschaft. Weitere personale Präferenzen (Gefühle, Familie, Religion u.a.) der Beteiligten könnten vernachlässigt werden. Bei diesen nichtethischen Theorien ist $\mathrm{zu}$ unterscheiden zwischen solchen, (1.) die den Menschen auf die Eigenschaften des Homo oeconomicus monologisch beschränken und solchen, (2.) die den Menschen mit einer egoistischen und einer sympathetisch-ethischen Rationalität dialogisch verstehen. Zur ersten Gruppe zählen ökonomisch imperialistische Ansätze, die in der Logik von Gary Becker und der Unternehmensberatung McKinsey o.a. unternehmerische Verantwortung als strukturelle Optimierungsprozesse von Organisationen verstehen, ohne den Aspekt der Menschendienlichkeit als eigenständigen Selbstzweck dagegen zu halten. Ansätze der zweiten Gruppe nicht-ethischer Führungstheorie verstehen den Menschen als ein dialogisches Wesen. Es geht ihnen womöglich auch um die Entfaltung des Menschen in dieser Qualität. Das Ziel der Menschendienlichkeit ist aber als sekundäres und austauschbares dem allein 
herrschenden Effizienzziel unterworfen und wird somit instrumentalisiert (Sims und Brinkmann 2003, S. 243). Bruno Frey vertritt eine solche Sicht der so genannten hellen Seite der Führung, nach der Moral eine ökonomische Erfolgsvariable ist: „Die Berücksichtigung der Sehnsüchte der Mitarbeiter bringt Wertschöpfung durch Wertschätzung" (Frey 2010, S. 643). Für Manfred Kets de Vries (2009) ist das Verstehen der Mitarbeiter eine Erfolgsstrategie, Zufriedenheit der Weg zu höherer Leistung. Stimmte diese eindeutig positive Synthese von Menschendienlichkeit und Effizienz, dürfte das humane Ziel trotz der Instrumentalisierung theoretisch nicht geopfert werden. Diese Grundannahmen zur normativen Wirksamkeit solcher Theorien sind aber keineswegs evident. Denn es gibt auch die dunkle Seite einer wirtschaftlich erfolgreichen Führung, die sich auch jenseits aller humanen Aspekte allein an der Effizienz orientiert. ${ }^{6}$ Nur solange die Berücksichtigung des humanen Ziels dem Ziel der Wirtschaftlichkeit dient, bleibt es legitim. Andernfalls kann und soll es sogar verworfen werden, um dann mit anderen Mitteln jenseits der Menschendienlichkeit das letztlich einzig geltende Ziel wirksam zu erreichen. Die persönliche Entfaltung des Menschen selbst ist in solchen Modellen also ein austauschbares Mittel zum Zweck der Effizienz. Zwar mögen die sichtbaren Resultate der konkreten Führungspraxis, die einer solchen Theorie folgt, zunächst auch normativ positiv empfunden werden. Doch ist die mitgedachte relativierende Verzweckung des Humanziels der Menschendienlichkeit das Ausschlusskriterium. Es handelt sich bei solchen Modellen deshalb nicht um Ethik. Sehr wohl aber zählen sie in den Bereich der Führungstheorie und sollten hier auch gebührend diskutiert werden.

Eine Führungsethik muss mit ihrer Wertebasis anders ansetzen: Hier wird neben der Wirtschaftlichkeit die Menschendienlichkeit als nicht instrumentalisierbarer Selbstzweck verstanden. ${ }^{7}$ Dies ist notwendig, denn die Entfaltung des Menschen als Person ist die unmittelbare Folge aus dem kategorischen Imperativ, den Menschen nie nur als Mittel, sondern immer zugleich als Zweck zu behandeln. Die Instrumentalisierung von Individuen für ökonomische Kalküle ist dann für eine Ethik verboten, selbst wenn dadurch Effizienz geschaffen würde. So geht es in der Führung als der wirksamen Beeinflussung von Individuen, Beziehungen und Regeln nicht allein um die Optimierung einer summierten Ausbringungsmenge, in der auch der eine oder andere

6 Solche Ansätze orientieren sich an der Logik von Machiavelli. Vgl. Kellermann (2004), S. 5. Allein der Erfolgsbezug ist hier das Unterscheidungskriterium zwischen vermeintlich starker und schwacher Führung.

7 Vgl. Ulrich (2010), S. 28; Kuhn und Weibler (2012), S. 23, 94, 107; Fischer und Fischer (2007), S. 22.
Mitarbeiter unter die Räder kommen könnte. Vielmehr ist ein wesentliches Führungsziel die persönliche Entwicklung jedes einzelnen Menschen in Abhängigkeit von fixen Grundfähigkeiten und variablen Persönlichkeitsmerkmalen (etwa Vorlieben). Damit darf das Effizienzziel aber nicht aus dem Blick geraten. Da Unternehmen in der Marktwirtschaft im Wettbewerb stehen, müssen individualethisch die Führungskräfte, wie institutionenethisch die Ausgestaltung der Regeln und Strategien, dabei immer auch den wirtschaftlichen Erfolg im Blick halten. Die Ausblendung der ökonomischen Sicht ist im marktwirtschaftlichen Kontext für Unternehmen vermutlich selbstdestruktiv. Es braucht unter dem Strich motivierte und gut qualifizierte Mitarbeiter, denen es nicht um Selbstverwirklichung auf Kosten des Unternehmens geht. Ansonsten besteht die Einladung zum Moral Hazard, was zusätzlich Identifikationspotenziale schwächt. Führungsethik muss also in ihrem Führungsverständnis bei allem notwendig geforderten Kalkül immer die Entfaltung jedes Mitarbeiters im Unternehmen als Selbstzweck normativ einfordern. Es sind alternative Gruppen einer Führungsethik zu unterscheiden.

1. Eine erste Schule legt der ökonomischen Wirtschaftsethik entsprechend eine Heuristik des Homo Oeconomicus zugrunde. Daraus lässt sich bei am Markt agierenden Unternehmen im Sinne der Effizienz eine Idee der Mitarbeiterschaft als Humanressourcen ableiten, die dennoch das Ziel der Menschendienlichkeit (eben im Sinne der Heuristik) nicht relativiert. Denn es dient das ökonomische Kalkül der Entfaltung des so kontrafaktisch modellierten Menschen. Die Effizienzorientierung tritt als Selbstzweck einer zuvor heuristisch vereinfachten Humanvorstellung als Ziel entgegen.

2. Alternativ kann eine komplexe anthropologisch begründete Menschennatur postuliert werden, die versucht den Menschen als Person auch jenseits der kontrafaktischen $\mathrm{HO}$-Heuristik in den Blick zu nehmen. Neben der Wirtschaftlichkeit soll

\begin{tabular}{|c|c|c|}
\hline & $\begin{array}{l}\text { Humanziel als Selbstzweck } \\
\text { (Ethik) }\end{array}$ & $\begin{array}{c}\text { Relativiertes } \\
\text { Humanziel } \\
\text { (keine Ethik) }\end{array}$ \\
\hline \multirow{2}{*}{$\begin{array}{l}\text { Komplexe } \\
\text { Anthropologie }\end{array}$} & $\begin{array}{l}\text { Personale Entfaltung als } \\
\text { Selbstzweck }\end{array}$ & \multirow{2}{*}{$\begin{array}{l}\text { Ökonomisch } \\
\text { instrumentalisierte } \\
\text { Entfaltung der } \\
\text { komplexen } \\
\text { Personalität }\end{array}$} \\
\hline & $\begin{array}{cc}\text { Deontologische } & \text { Metaphysische } \\
\text { Ansätze } & \text { Ansätze }\end{array}$ & \\
\hline $\begin{array}{l}\text { Einfache } \\
\text { Heuristik }\end{array}$ & $\begin{array}{l}\mathrm{HO} \text {-Entfaltung als Selbstzweck } \\
\text { in normativ individualistischen } \\
\text { Ansätzen }\end{array}$ & $\begin{array}{l}\text { Ökonomischer } \\
\text { Imperialismus }\end{array}$ \\
\hline
\end{tabular}

Tabelle 3.1 Menschendienlichkeit in Führungstheorie und Führungsethik

Quelle: Eigene Darstellung 
die Führungskultur dieser personalen Entfaltung des Menschen dienen, weil diese Ethikmodelle nicht in der sie endogenisierenden Ökonomik aufgehen. Die Optimierung des Menschen in seiner Entfaltung als Person in Abhängigkeit von fixen und variablen Persönlichkeitsfaktoren muss also unbedingt mitgedacht werden. Dann ist im Sinne der Menschendienlichkeit die personale Entfaltung in dieser Komplexität der Selbstzweck, der dem Wirtschaftlichkeitsziel entgegentritt. Es sind hier wiederum deontologische Ansätze kantischer Prägung und metaphysisch (religiös) begründete Modelle zu unterscheiden.

\subsection{Zweiter Schritt: Heuristische oder anthropologische Wertebasis?}

Es wurde gezeigt, warum einfache Heuristik und komplexe Anthropologie als zwei konkurrierende Paradigmen der Führungsethik Sozialer Marktwirtschaft anerkannt und unterschieden werden müssen. Der zweite Schritt muss nun in der zu prüfenden Führungsethik diese notwendige Zuordnung identifizieren, auch um über die weitere Anwendung von LIFESMA zu entscheiden.

Monologische Modelle der Ökonomik sehen in dem am Markt agierenden Menschen heuristisch den zweckrationalen Homo Oeconomicus. Danach sollen Menschen dieser Logik sowohl in der Gestaltung der Regeln eines Unternehmens wie in den konkreten Spielzügen im Unternehmen folgen. Eine davon abweichende Moral ist überflüssig und sogar schädlich (Homann und Blome-Drees 1992). Das Humanziel ist hier in das Effizienzziel endogenisiert, so dass die Synthese für den Verantwortungsdualismus identisch ist mit einer ökonomisch begründeten Verantwortung. Der bewusst dafür in Kauf genommene Preis ist die auch von Ökonomen als realitätsfern eingeschätzte Verkürzung des Menschen aus heuristischen Gründen. ${ }^{8}$ Vor allem ökonomische Theorien der Führungsethik etwa aus dem Schülerkreis Homanns sind diesem heuristischen Paradigma zuzuordnen.

8 So schließt sich der Governance-Theoretiker Tricker (2012, S. 62) der Fundamentalkritik hinsichtlich des dem ökonomischen Ansatz nahe stehenden Agency-Ansatzes an: „agent theory argues that it has been erected on a single, questionable abstraction that governance involves a contract between two parties, and is based on a dubious conjectural morality that people maximize their personal utility. " Die Volkswirte Stutzer et al. (2010) konstatieren in einem nicht veröffentlichten DFG-Antrag: „Nicht nur methodisch, auch inhaltlich zeichnet sich ein Paradigmenwechsel ab. [...] Auch die Ergebnisse der experimentellen Wirtschaftsforschung stellen eine Herausforderung der Mainstream-Ökonomie dar. Sie zeigen dabei altruistische und gerechtigkeitsgeleitete Verhaltensweisen, welche mit dem Paradigma des $\mathrm{HO}$ nicht ohne weiteres vereinbar scheinen."
Eine komplexe Anthropologie kann sich auf Smith (1926/1994, S. 1) berufen: „Mag man den Menschen für noch so egoistisch halten, es liegen doch offenbar gewisse Prinzipien in seiner Natur, die inn dazu bestimmen, an dem Schicksal anderer Anteil zu nehmen, und die inm selbst die Glückseligkeit dieser anderen zum Bedürfnis machen."

Das Humanziel der Ethik kann sich unter dieser Voraussetzung nicht monologisch auf eine Entfaltung und Förderung egoistischer Eigennutzenmaximierung beschränken, weil diese Reduzierung den Menschen in der Ausblendung altruistischer oder auch deontologischer Rationalität (Sen 1993/2002, S. 31) verkürzt und die Umsetzung einer solchen Ethik dem Menschen nicht gerecht wird. Wird diese Anthropologie der Heuristik vorgezogen, so ist es die Aufgabe einer dem Humanziel verpflichteten Führung, beide Wesensanlagen gleichermaßen ernst zu nehmen und Menschen als in dieser Weise dialogische Wesen zu verstehen. Führungsethiken etwa, die den anthropologischen Annahmen dialogischer Rationalität von Ulrich (2008) folgen, sind diesem Paradigma zuzuordnen.

Mit der sorgfältigen Einordnung des untersuchten Ansatzes der Führungsethik in eines der beiden Paradigmen ist mithilfe der LIFESMA-Wertebasis der zweite Schritt getan, bevor die Einzelbewertungen folgen. Diese können nun für das anthropologische Paradigma durchgespielt werden.

\section{Anwendung mithilfe der Prinzipien und Perspektiven}

\subsection{Dritter Schritt: Einzelbewertungen}

In einem dritten Schritt wird versucht, aus den drei ethischen Perspektiven jeweils die Intensität der drei Kulturprinzipien in der zu prüfenden Ethik zu identifizieren. Es folgt dabei als Orientierung für die konkrete Anwendung von LIFESMA eine exemplarische Vorstellung führungsethisch relevanter Inhalte für die Bewertung, gegliedert nach den drei Kulturprinzipien.

\subsubsection{Vertrauenskultur}

Die Führungstheorie unterscheidet seit McGregor (1960) hinsichtlich des Humanverständnisses der Führungskraft $z$ wischen einer Theorie $X$ und einer Theorie $Y$ mit entsprechenden Konsequenzen für die Gestaltung der Vertrauenskultur.

Dem anthropologisch dialogischen Paradigma widerspricht die Theorie X, da der Mensch dort im Kontext der Unternehmung nicht als moralisches Wesen 
verstanden werden muss. Diese Theorie sei hier als Folie eines Gegenmodells vorgestellt: Der Mitarbeiter gilt als opportunistisch und egoistisch. Da er jede Gelegenheit zum Moral Hazard nutzt, was dem Unternehmen schadet, bedarf es institutionenethisch eines intensiven Kontrollsystems mit entsprechenden Sanktionsinstrumenten (McGregor 1960, S. 33-35). Führung ist dann im Sinne der Kategorisierung von MacGregor Burns (1978) und der Weiterführung von Bass (1985) transaktional zu verstehen. Das heißt: Da Menschen im Unternehmen nicht moralisch konvertierbar sind und somit eine entsprechende Umerziehung als utopisch gilt, müssen sich beziehungsethisch die Individuen zu den Regeln so verhalten, dass opportunistische Mitarbeiter sich zumindest an das von innen Erwartete anpassen, während es nicht auf geteilte Ideale oder Visionen ankommt (MacGregor Burns 1978, S. 20). Transaktionale Führung basiert auf Kontrolle, extrinsischer Belohnung und Sanktionierung: „Für das, was sie tun oder lassen, haben die Geführten mit positiven oder negativen Konsequenzen zu rechnen, die die Führungskraft vermittelt“ (Neuberger 2002, S. 197). Controlling soll dabei vor allem opportunistisches Verhalten reduzieren. Die Führungskraft muss gegenüber den Mitarbeitern die Spannung von Macht und Gegenmacht erfolgreich gestalten und sich in diesem Gegeneinander behaupten. Anpassungsfähigkeit wie der Respekt vor vorgegebenen Regeln und Sanktionen ist ein Einstellungskriterium für Führungskräfte und Mitarbeiter. Nicht leitende Mitarbeiter sollen nicht nur den Regeln, sondern auch der Führungskraft vertrauen. Weniger gefordert sind hier Kreativität oder Diskussionsfreudigkeit, weil diese die Umsetzung von Entscheidungsprozessen blockieren und das vorausgesetzte Prinzipal-Agenten-Verhältnis unterminieren.

"The Kantian adopts the Theory $Y$ view of human nature" (Bowie 1999, S. 86). Kantische und überhaupt anthropologisch-dialogische Führungsethik setzt auf eine Kultur des Vertrauens und der Wertschätzung als Selbstzweck. Danach wird im Sinne des StewardshipAnsatzes (vgl. etwa Tricker 2012) zunächst davon ausgegangen, dass Mitarbeiter loyal, arbeitsbereit und motiviert sind. Sie wollen Verantwortung und Kreativität entfalten. Es ist dann in einer Unternehmenskultur gegenseitigen Vertrauens mehr auf Selbstkontrolle zu setzen (McGregor 1960, S. 47 f.). Institutionenethisch dienen die Regeln dieser Kultur. Sie ersetzen nicht die Individualmoral der Akteure. Eine solche Theorie kann beziehungsethisch in einer transformationalen (bzw. transformativen) Führung umgesetzt werden. Sie baut auf Intervention zum Zweck der Identifikation etwa mit Vision und Mission, etwa durch Charisma, Inspiration, intellektuelle Stimulation oder individualisierte Führung
(Neuberger 2002, S. 199). Sie realisiert sich individualethisch entweder durch ein charismatisch-heroisches Führungsverständnis, nach dem Mitarbeiter zu erziehen sind; oder aber durch ein post-heroisches Führungsverständnis, nach dem eher stille Leader partizipativ Entscheidungen treffen (Kuhn 2000, S. 160). Deren Ziel ist die möglichst umfassende Übernahme von Mitverantwortung bei angstfreier kritisch-kreativer Loyalität.

\subsubsection{Verantwortungskultur}

Die Führungsethik muss eine der Wertebasis entsprechende Begründung der Verantwortung anbieten. Dazu seien als Grundlage individualethischer Führungstugend hier exemplarisch eine kantische und eine christliche Variante vorgestellt. ${ }^{9}$ Ethisch legitime Führung geht im kantischen Sinne aus von autonomen Führungskräften, die in der Verantwortung vor dem Sittengesetz ihre Mitarbeiter zur Autonomie befähigen: „... one has both a duty of perfection to oneself and a duty to promote the happiness of others" (Bowie und Werhane 2005, S. 64). Zuerst ist der Mensch in dieser deontologischen Führungsethik hier verantwortlich vor den Denknotwendigkeiten der Vernunft, daraus abgeleitet vor sich selbst, die eigene Autonomie zur Entfaltung zu bringen und schließlich vor den Mitarbeitern, ihnen zu helfen, ihre Autonomie zu entfalten. Eine Priorität des Humanen gilt ebenso für eine christliche Führungsethik: Danach ist der Mensch als Freiheits- und Sozialwesen moralische Existenz, die in Entscheidungen grundsätzlich dialogisch Eigennutzenorientierung und Sozialorientierung mithilfe des Gewissens zu Entscheidungen überführt. Aus christlicher Sicht trägt der Mensch im Sinne des dreifachen biblischen Liebensgebots eine dreifache Verantwortung zuerst gegenüber dem Schöpfer, der dem Menschen die Freiheit der Selbstbestimmung und den Auftrag zum Guten mitgegeben hat. Daraus abgeleitet hat er gegenüber sich selbst den Auftrag zur Entfaltung seiner individuellen und sozialen Personalität und zur Wertschätzung jedes menschlichen Lebens, gerade auch des schwachen. Drittens ist die Verantwortung gegenüber den Mitmenschen ein Auftrag der Nächstenliebe, die sich beziehungsethisch in einem auch affektiven Geist des Miteinanders realisiert. Kantisch wie christlich haben Führungskräfte also eine dreifache Verantwortung. Institutionenethisch sollen die Regeln zur Einlösung des Humanziels die Entfaltung dieser Verantwortung ermöglichen (etwa durch gezielte Fortbildungen, flache Hierarchien, Delegation,

9 Passend wären hier etwa auch Begründungen im Sinne der Business Metaphysics (Schramm 2015) oder aristotelische Ableitungen. 
kooperative Entscheidungen, Einstellung entsprechender Mitarbeiter).

Partizipative Führung wird dabei nicht als Konsensdemokratie verstanden. Vielmehr ist es etwa in kantischem Sinne der Autonomie der Individuen geschuldet, dass es unterschiedliche Interessen gibt, die auch durch Diskurse nicht immer zu einem Konsens geführt werden können. Aufgabe der Führungskraft ist es, den Ausgleich der unterschiedlichen Interessen zu gestalten und dabei auch Differenzen auszuhalten: „We should prefer a theory that leadership that allows a place of disagreement and dissent" (Bowie und Werhane 2005, S. 142). Dazu gehört auch eine überzeugende Durchsetzungskompetenz (Ebd., S. 147). Beziehungsethisches Ziel ist eine Symbiose aus Effizienz- und Humanziel durch verändertes habituelles Denken und Handeln: „Diese Führung erfolgt, wenn eine oder mehrere Personen sich so miteinander verbinden, dass Führer und Gefolgsleute einander zu höheren Niveaus von Motivation und Moralität emporheben“ (Mac Gregor Burns 1978, S. 20). Postheroische Führungskräfte etwa begegnen den Mitarbeitern als selbständigen Mitverantwortlichen. Erfolg versprechende Aufgaben sind in der Regel zu delegieren, während - soweit möglich - Verantwortung für Fehler der Geführten übernommen wird (Kuhn 2000, S. 160).

Transformationale Führung zeigt sich darin, dass sie die Mitarbeiter zu autonomen Mitverantwortlichen konvertieren will. Auch die Geführten führen die Führungskraft durch ihr Verhalten und werden so als Mit-führende verstanden. Die Befähigung dazu erfordert die Transparenz von Entscheidungswegen und -argumenten ebenso wie die gegenseitige Bereitstellung aller notwendigen Informationen für rationale mitverantwortliche Entscheidung (open book management) (Bowie 1999, S. 54-57).

Gegebenenfalls kann Eigenverantwortung im Sinne der Autonomie so weit interpretiert werden, dass intrinsische die extrinsische Motivation der Mitarbeiter ersetzen soll. Reinhard Sprenger etwa stellte Ende der Neunziger Jahre in seinen narrativen Bestsellern die Erfolgsrezepte eigenverantwortlicher Führung heraus und warnte vor zu viel Mitarbeiterlob im Unternehmen, eine zumindest beziehungsethisch durchaus streitbare Empfehlung.

\subsubsection{Befähigungskultur}

Notwendige Bedingung für eine Kultur der Befähigungsfreiheit ist die unbedingte Pflicht zur Garantie negativer Freiheit als „ability to act independently of determination by alien causes" (Bowie 1998, S. 1085). Zwang und Täuschung sind unbedingt zu vermeiden. ${ }^{10}$ Auch

10 Vgl. Korsgaard (1996), S. 113. Eine betriebsbedingte Kündigung ist dann deshalb legitimierbar, wenn die Mitarbeiter sich in einem ein Paternalismus, der den Mitarbeitern eine Idee guten Lebens vorschreibt, ist unerwünscht (Bowie 1999, S. 71) Weiteres Ziel sinnvoller Arbeit ist im Sinne dieser deontologischen Führungsethik die Förderung der Entfaltung positiver Freiheit "to be a law unto themselves“ (Ebd., S. 63). Dazu sind etwa durch Bildungsangebote einerseits Optionsräume für freie Entscheidungen (inklusive Exit-Optionen) auszuweiten. Andererseits sollen die rationale Entscheidungsfähigkeit und die moralische Entwicklung gefördert werden, um so Mitarbeiter zur personalen Entfaltung positiver Freiheit zu befähigen (Ebd., S. 70). Dazu zählt die Förderung der Integrität von Führungskräften und Mitarbeitern durch das Verstehen ihrer Aufgaben. ${ }^{11}$ Dabei handelt es sich um eine über das wertneutral Charismatische hinausgehende, Orientierung gebende Selbstbestimmung. Integrität gilt als eindeutig positive Wertbindung, die sich in persönlicher Kohärenz und Standhaftigkeit auch gegen Widerstände realisiert (Kuhn und Weibler 2012, S. 111113). Führungskräfte haben dabei im Sinne des Prinzips ultra posse nemo tenetur in ihrem konkreten Einsatz zur Förderung positiver Freiheit selbst einen Optionsraum, welche Maßnahmen sie für sinnvoll halten. Verschiedene Führungsethiken versuchen eine entsprechende Umsetzung.

Kantische und ebenso christliche Führungsethik sind institutionenethisch Regel- und Strategiekonzepte einer Befähigungsfreiheit der Hilfe zur Selbsthilfe. Objektiv verstandener Selbstzweck ist die Befähigung des Menschen zur Übernahme der jeweiligen dreifachen Verantwortung. Sie wird konkret in den Regeln, die als Garanten positiver Freiheit dazu befähigen. Daneben ist die individualethische Entfaltung nichtegoistischer Tugend in allen Lebensbereichen Ausdruck dieser Freiheit, aus der Leistung, Motivation, Loyalität und Hilfsbereitschaft folgen. Im Sinne eines Modells beidhändiger Führung ist darauf zu achten, dass die Menschen mit dynamisch-kreativen Potentialen zu innovativem, andere wiederum eher zu einer Verstetigung bekannter Arbeitsprozesse zu motivieren sind. ${ }^{12}$ Sowohl die Gestaltung von Regeln und Strategien im Unternehmen wie die konkreten Spielzüge sind hierzu systematische Orte der Moral. ${ }^{13}$

freiwilligen Arbeitskontrakt auf dieses Risiko eingelassen haben. Vgl. dazu auch Bowie (1999), S. 48-53.

11 Integrität zeigt sich etwa in fachlicher Qualifikation, in der Erfahrung im Umgang mit Menschen, in einer stimmigen Persönlichkeitsstruktur, die sich an begründeten Werten orientier, Emotionen zeigt und positive Tugenden lebt.

12 Vgl. etwa Müller et al. (2015) und Jansen et al. (2009) zu diesem Modell des Ambidextrous Leadership mit exploitativen und explorativen Anreizen.

13 Vgl. Schockenhoff (2007), S. 113: „Gerechtigkeitsfragen können im Blick auf die motivationale Verfasstheit der Personen erörtert 
Servant Leadership versteht Befähigung im Sinne des postheroischen Ideals. ${ }^{14}$ Inspiriert durch Hesses „Morgenlandfahrt" entwarf der Unternehmer Greenleaf (1904-1990) die Vision einer dienenden Grundhaltung von Führungskräften. Durch das individualethisch eingeforderte dienende Selbstverständnis der Führungskraft sollen beziehungsethisch die Mitarbeiter zur Eigen- und Mitverantwortung inspiriert und befähigt werden. Evangelikale Weiterentwicklungen wie die von Blanchard, Hodges oder Jennings stellen ein solches Führungsverständnis unter das Motto Lead like Jesus. Hier werden mit intuitiven Bibelbezügen Grundsätze und Tugenden aneinandergereiht, wie Heart, head, hands and habit, womit die rechte Motivation, das Vorhandensein einer Vision wie ein entschlossenes Handeln mit spirituellem Charisma gemeint sind; oder etwa die Tugenden des Wegbereiters, des visionären Leistungsmotivators o.a. Die säkulare Version von Sipe und Frick bietet eine Matrix mit 21 Führungskompetenzen, die in 7 Kategorien eingeteilt sind (Kommunikator, Teamarbeiter, moralische Autorität, systematischer Denker, Charakterstärke, Weitblick, den Menschen zuerst in den Blick nehmen).

Auch die anthroposophische Führungsethik, die Werner mit der Drogeriemarktkette $d m$ auf der Grundlage eines weltanschaulich transparent gemachten Humanzieles konsequent umsetzt (Dietz 2007), vertritt einen starken Befähigungsgedanken. Ausgehend von der individualethisch zu verinnerlichenden Idee einer irdisch aufgegebenen Selbsterlösung des Menschen geht es darum, beziehungsethisch die Führungskräfte zu Evokatoren der jedem Menschen innewohnenden Kreativität zu machen. Mitarbeiter sind dazu zu befähigen, innere Ausgeglichenheit zu finden, indem sie die in ihnen wohnenden Unternehmertalente entdecken und entfalten. Eine solche Kultur gegenseitiger Befähigung führe als Symbiose von Effizienz- und Humanziel zum Mitunternehmertum, zu einer hohen Identifikation mit sich und dem Unternehmen verbunden mit einem affektiven Zusammengehörigkeitsgefühl. Der wirtschaftliche Erfolg ist als abgeleitetes Ziel zu verstehen, dem die so genannte Veredelung jedes Menschen nicht geopfert werden darf. Auf dieser Grundlage kann Werner (2006, S. 29) für eine am anthroposophischen Menschenbild stimmig ausgerichtete Führungsethik das transformationale Ziel der Befähigungskultur so beschreiben: „Zwischen der Durchführung einer Weisung und einem Handeln aus einer Einsicht ist von außen kaum

werden, die im horizontalen Rahmen [...] oder innerhalb vertikaler Verantwortungsbeziehungen zu gerechtem Handeln aufgerufen sind."

14 Vgl. Hartmann (2013), S. 23-65 für eine Übersicht. ein Unterschied zu sehen. Auf diesen Unterschied aber kommt es entscheidend an."

\subsection{Vierter Schritt: Gesamtbewertung}

Nach der Anwendung von LIFESMA liegen im besten Fall neun Einzelbewertungen zur Übereinstimmung mit den Grundideen Sozialer Marktwirtschaft vor. Dabei wird es in der konkreten Prüfung von Modellen der Führungsethik vermutlich in den Bewertungsfeldern auch Lücken geben, wenn identifizierbare Antworten zu einzelnen Feldern fehlen. Zu beachten ist, ob insgesamt ein Prinzip oder eine Perspektive komplett ausgeblendet wird. In einem solchen Fall müsste eine Gesamtbewertung dies kritisch anmerken. Die Einzelbewertungen müssen nun noch einmal hinsichtlich ihrer Wechselwirkung auf andere Kulturprinzipen befragt werden. Z.B. ist eine beziehungsethisch möglicherweise positiv bewertete Befähigungskultur eines Servant Leadership-Modells mit einer dadurch bedingten möglichen Gefährdung für die Autonomie der Führungskräfte durch eine überfordernde Selbstaufgabe vor allem gegenüber der Organisation zu konfrontieren (im Feld: individualethische Eigenverantwortung), wie sie etwa Bowie und Werhane (2005), S. 143 äußern: "The danger of the servant leader is that he or she should allow him- oder herself to be used as a means merely“. Analoges gilt etwa für ein anthroposophisches Modell transaktionaler Führung mit seinen möglichen paternalistischen Konsequenzen.

Anschließend ist eine Gewichtung der Einzelbewertungen vorzunehmen. Daraus ergibt sich dann die Bewertung als starke oder schwache Theorie Sozialer Marktwirtschaft und eine entsprechende Empfehlung hinsichtlich ihrer Anwendung in Unternehmen vor dem Hintergrund, so Soziale Marktwirtschaft neu zu kultivieren.

\section{Ausblick}

LIFESMA identifiziert die Grundfrage der Führungsethik Sozialer Marktwirtschaft im Verantwortungsdualismus von Effizienz- und Humanziel. Damit ist ein Schritt getan, wie eine Kongruenz ethischer Grundprinzipien auf Makro- und Mikroebene der Wirtschaft gestärkt werden kann: Das vorgestellte Modell hilft als Leitfaden grundsätzlich Führungsethiken von anderen Führungstheorien zu unterscheiden. Das anthropologische (deontologisch oder religiös metaphysisch begründete) Paradigma bietet dazu einen Filter für die Bewertung von entsprechenden Führungsethikkonzepten.

Es tun sich auf dieser Grundlage weiterführende Forschungsfragen auf. So kann über die hier 
vorgenommenen Komplexitätsreduzierungen (wie etwa eine mögliche Ergänzungsbedürftigkeit der Kulturprinzipien) diskutiert werden, ebenso über den hier starken kantischen Einschlag (Bowie), den man teilen mag oder nicht. Eine christliche Bereicherung halte ich für möglich und lohnend, sobald eine umfassende Systematik christlicher Führungsethik vorliegt. ${ }^{15}$

Ein analoger Leitfaden für das alternative heuristische Paradigma steht aus. Er kann sich grundsätzlich an dem vorgelegten Modell orientieren, geht es

15 Für Schritte auf diesem Weg vgl. Nass (2014); ders. (2015); ders. (2017).

\section{Literaturverzeichnis}

Battyány, Philip (2007), Zwang als Grundübel in der Gesellschaft? Der Begriff des Zwangs bei Friedrich August von Hayek, Tübingen.

Bass Bernhard (1985), Leadership and Performance beyond Expectations, New York.

Becker, Manfred (2002), Personalentwicklung: Bildung, Förderung und Organisationsentwicklung in Theorie und Praxis, Stuttgart.

Bowie, Norman (1998), A Kantian Theory of Meaningful Work, Journal of Business Ethics 17, S. 1083-1092.

Bowie, Norman (1999), Business Ethics. A Kantian Perspective, Oxford.

Bowie, Norman und Patricia Werhane (2005), Management Ethics, Oxford.

Dietz, Karl-Martin (2007), Die ontologische Grundlage der Autonomie des Menschen und ihr Rang in der modernen Führungspraxis, in: Waldemar Schreckenberger (Hg.), Recht, Staat und kulturelle Entwicklung, Speyerer Arbeitsheft Nr. 191, Speyer, S. 97-109.

Eucken, Walter (1952/2004), Grundsätze der Wirtschaftspolitik, 7. Aufl., Tübingen.

Fischer, Lorenz und Oliver Fischer (2007), Sind zufriedene Mitarbeiter gesünder und arbeiten härter? Personalführung 40 (3), S. 20-32.

Frey, Bruno F. et al. (2010), Unternehmenserfolg durch ethikorientierte Unternehmens- und Mitarbeiterführung. In: Uto Meier und Bernhar Sill (Hg.), Führung, Macht, Sinn, Regensburg, S. 637-656.

Goldschmidt, Nils (2006), Walter Eucken: Grundsätze der Wirtschaftspolitik (1952), in: Dietmar Herz und Veronika Weinberger (Hg.), Lexikon der ökonomischen Werke, Düsseldorf, S. 129-130.

Hartmann, Mathias (2013), Servant Leadership in diakonischen Unternehmen, Stuttgart.

Hartshorne, Charles (1948/1964), The Devine Relativity. A Social Conception of God, New Haven - London. doch auch hier um eine Identifizierung von Führungsethik Sozialer Marktwirtschaft. Bei den Kulturprinzipien ist dabei eine Überarbeitung der Befähigungsfreiheit, bei den Perspektiven der Individualethik zu erwarten. Anschließend ließe sich damit ein analoges Prüfverfahren von Führungsethiken im Kontext jenes Paradigmas durchführen. Auch könnten mit einem solchen zweiten Filter dann Modelle, deren Zuordnung nicht eindeutig ist, von beiden Leitfäden bewertet werden, was einen ertragreichen Dialog der jeweiligen Ergebnisse verspricht und so die Weiterentwicklung systematischer Führungsethik bereichert.
Hegner, Jan (2000), Alexander Rüstow. Ordnungspolitische Konzeption und Einfluß auf das wirtschaftspolitische Leitbild der Nachkriegszeit in der Bundesrepublik Deutschland, Stuttgart.

Homann, Karl und Franz Blome-Drees (1992), Wirtschafts- und Unternehmensethik, Göttingen.

Homann, Karl (1993), Die Funktion der Moral in der modernen Wirtschaft, in: Josef Wieland (Hg.), Wirtschaftsethik und die Theorie der Gesellschaft, Frankfurt a.M., S. 32-53.

Jansen, Justin J.P., Dusya Vera und Mary Lou Crossan (2009), Strategic leadership for exploration and exploitation: The moderating role of environmental dynamism, The Leadership Quaterly 20 (2009), S. 5-18.

Kellermann, Barbara (2004), Bad Leadership. What it is, how it Happens, why it Matters, Boston.

Kets de Vries, Manfred (2009), Führer, Narren und Hochstapler. Die Psychologie der Führung, Stuttgart.

Korsgaard, Christine (1996), Creating the Kingdom of Ends, New York.

Kreuer, Ellen K. (2017), Gerechte Mitarbeiterführung: Eine unternehmensethische Analyse. Nicht veröffentlichte Bachelorarbeit an der wirtschaftswissenschaftlichen Fakultät der Westfälischen Wilhelms-Universität Münster.

Kuhn, Thomas (2000), Internes Unternehmertum. Begründung und Bedingungen einer ,kollektiven Kehrtwendung', München.

Kuhn, Thomas und Jürgen Weibler (2012), Führungsethik in Organisationen, Stuttgart.

MacGregor Burns, James (1978), Leadership, New York. McGregor, Douglas (1960), The Human side of Enterprise, New York.

Melé, Domenec und César G. Cantón (2014), Human Foundations of Management. Understanding the Homo Humanus. Palgrave Macmillan. New York. 
Müller, Christian und Elmar Nass (2013), Normative Grundlagen des Ordoliberalismus, in: Herbert Pribyl (Hg.): Die Weltwirtschaftskrise. Lösungsansätze aus christlich-ethischer Sicht, Heiligenkreuz: Sarto, S. 157-183.

Müller, Julia, Matthias G. Will und Barbara Renzl (2015), Ambidextrous Leadership and a Micro-MacroModel for Systemizing Research of a Multi-LevelPhenomenon. Paper presented at the SMS $35^{\text {th }}$ Annual International Conference $6^{\text {th }}$ October 2015, Denver.

Nass, Elmar (2014), Christliche Personalführung. Ein Führungsmodell (nicht nur) für Diakonie und Caritas, Zeitschrift für Marktwirtschaft und Ethik 2/2014, S. 3-21.

Nass, Elmar (2015), A Christian Theory of Leadership Ethics, in: Catholic Social Science Review 20 (2015), S. 3-19.

Nass, Elmar (2017), Handbuch Führungsethik, Band 1: Grundlagen: Systematik und Denkrichtungen, Stuttgart.

Neuberger, Oswald (2002), Führen und führen lassen, Stuttgart.

Nussbaum, Martha (1988), Die Natur des Menschen, seine Fähigkeiten und Tätigkeiten: Aristoteles über die distributive Aufgabe des Staates, in: dies. (1999), Gerechtigkeit oder Das gute Leben, Frankfurt a.M., S. 86-130.

o.V.: Die wichtigste Ressource, in: Handelsblatt vom 19.5.2005 http://www.handelsblatt.com/unternehmen/ management/strategie/vier-deutsche-unternehmensind-unter-den-top-ten-managerschmieden-europasbmw-lufthansa-voith-und-degussa-die-wichtigsteressource/2552920.html (19.9.2017).

Plaschke, Jürgen, Werner Sauter und Thomas G. Zinder (2007), Personalmanagement, Berlin.

Rodek, Hans-Georg (2012): Die Ressource Mensch muss optimiert werden, in: Die Welt vom 13.4.2012, http://www.welt.de/kultur/kino/article106173907/ Die-Ressource-Mensch-muss-optimiert-werden. html (19.9.2017)

Rohrhirsch, Ferdinand (2013), Christliche Führung Anspruch und Wirklichkeit. Führen mit Persönlichkeit und Ethik, Berlin.

Schockenhoff, Eberhard (2007), Grundlegung der Ethik, Freiburg i.Br.
Schramm, Michael (2015), Die Ethik der Transaktion. Warum eine Business Metaphysics im operativen Management nützlich ist, in: Matthias Maring (Hg.), Vom Praktisch-Werden der Ethik in interdisziplinärer Sicht: Ansätze und Beispiele der Institutionalisierung, Konkretisierung und Implementierung der Ethik. Schriftenreihe des Zentrums für Technik- und Wirtschaftsethik am Karlsruher Institut für Technologie, Band 7, Karlsruhe, S. 173-191.

Sen, Amartya (1993), Capability and Well-Being, in: Martha Nussbaum und Amartya Sen (Hg.) (2002), The Quality of Life, Oxford: S. 30-53.

Sen, Amartya (2003), Commodities and Capabilities, New Delhi.

Sims, Ronald R. und Johannes Brinkmann (2003), Enron Ethics (or: Why Culture Matters more than Codes), Journal of Business Ethics 45 (3), S. 243-256

Smith, Adam (1926/1994), Theorie der ethischen Gefühle, Hamburg.

Stutzer, Alois et al. (2010), Normative und positive Grundlagen der Glücksforschung. Antrag zu einem bei der DFG eingereichten Forschungsprojekt, Münster/Basel (nicht veröffentlicht).

Ulrich. Peter (1999). Führungsethik. In: W. Korff u.a. (Hg.): Handbuch der Wirtschaftsethik, Bd. 4: Ausgewählte Handlungsfelder, Gütersloh, S. $230-248$.

Ulrich, Peter (2008), Integrative Wirtschaftsethik: Grundlagen einer lebensdienlichen Ökonomie, Bern u.a.

Ulrich, Peter (2010), Zivilisierte Marktwirtschaft. Eine wirtschaftsethische Orientierung, Bern u.a.

Tricker, Bob (2012), Corporate Governance. Principles, Policies and Practices, Oxford.

von Oelsnitz, Dietrich (2012), Einführung in die systemische Personalführung, Heidelberg.

Werner, Götz (2006), Führung für Mündige. Subsidiarität und Marke als Herausforderungen einer modernen Führung, Karlsruhe.

Whitehead, Alfred N. (1929/1978), Process and Reality. An Essay in Cosmology. Gifford Lectures Delivered in the University of Edinburgh 1927-28, edited by David R. Griffin und Donald W. Sherbume, New York/London. 
